\title{
Article
}

\section{Intelligent Agents in Co-Evolving Knowledge Networks}

\author{
Evangelos Ioannidis $^{1, *}$, Nikos Varsakelis ${ }^{2}$ and Ioannis Antoniou ${ }^{1}$ \\ 1 Complex Systems Analysis Laboratory (COSAL), Faculty of Sciences, School of Mathematics, \\ Aristotle University of Thessaloniki, 54124 Thessaloniki, Greece; iantonio@math.auth.gr \\ 2 Complex Systems Analysis Laboratory (COSAL), Faculty of Economic and Political Sciences, \\ School of Economics, Aristotle University of Thessaloniki, 54124 Thessaloniki, Greece; barsak@econ.auth.gr \\ * Correspondence: ioannidek@math.auth.gr
}

check for

updates

Citation: Ioannidis, E.; Varsakelis, N.; Antoniou, I. Intelligent Agents in Co-Evolving Knowledge Networks. Mathematics 2021, 9, 103. https:// doi.org/10.3390/math9010103

Received: 11 December 2020 Accepted: 29 December 2020 Published: 5 January 2021

Publisher's Note: MDPI stays neutral with regard to jurisdictional clai$\mathrm{ms}$ in published maps and institutional affiliations.

Copyright: () 2021 by the authors. Licensee MDPI, Basel, Switzerland. This article is an open access article distributed under the terms and conditions of the Creative Commons Attribution (CC BY) license (https:// creativecommons.org/licenses/by/ $4.0 /)$.

\begin{abstract}
We extend the agent-based models for knowledge diffusion in networks, restricted to random mindless interactions and to "frozen" (static) networks, in order to take into account intelligent agents and network co-evolution. Intelligent agents make decisions under bounded rationality. This is the key distinction of intelligent interacting agents compared to mindless colliding molecules, involved in the usual diffusion mechanism resulting from accidental collisions. The co-evolution of link weights and knowledge levels is modeled at the local microscopic level of "agent-to-agent" interaction. Our network co-evolution model is actually a "learning mechanism", where weight updates depend on the previous values of both weights and knowledge levels. The goal of our work is to explore the impact of (a) the intelligence of the agents, modeled by the selection-decision rule for knowledge acquisition, (b) the innovation rate of the agents, (c) the number of "top innovators" and (d) the network size. We find that rational intelligent agents transform the network into a "centralized world", reducing the entropy of their selections-decisions for knowledge acquisition. In addition, we find that the average knowledge, as well as the "knowledge inequality", grow exponentially.
\end{abstract}

Keywords: networks; knowledge diffusion; co-evolution dynamics; agent-based modeling; computer simulations; bounded rationality; intelligent agents; entropy; network centralization; innovation rate

\section{Introduction}

The increasing complexity of the highly interconnected 21st century economy [1-4] is modeled in terms of knowledge networks [5-7]. The emerging dynamics of knowledge in complex networks depends on 11 Conditioning Factors (CF) [8-11]:

(CF1) The structure of the network;

(CF2) The evolutionary dynamics of the network;

(CF3) The presence of agents creating knowledge (innovative agents);

(CF4) The initial knowledge of the agents;

(CF5) The presence of agents with false beliefs (negative knowledge level);

(CF6) The presence of unreliable communication channels (negative weights);

(CF7) The selection rule (\$) of agents for communication and knowledge acquisition;

(CF8) The filtering rule $(\mathbb{H})$ of agents for excluding other agents for knowledge acquisition;

(CF9) The order of implementation of the selection rule (CF7) and the filtering rule (CF8) by the agents of the network;

(CF10) The attacks at nodes or links;

(CF11) The position of experts (highly knowledgeable agents) within the network.

The dependence on network structure (CF1) was studied for regular, random, and smallworld networks [12-14], as well as for scale-free and hierarchal networks [15-17]. The dependence on network evolution (CF2) was studied for networks with probabilistic edgerewiring [18-21]. The presence of agents creating knowledge (CF3) increases significantly the speed of knowledge growth [22,23]. However, the knowledge stock is rising with 
a high level of disparity for different knowledge levels. The dependence on the initial knowledge levels (CF4) has been addressed, revealing that agents with low initial knowledge may outperform agents with higher initial knowledge [8]. The presence of initial false beliefs (CF5) and unreliable communication channels (CF6) has been addressed, revealing that initial false beliefs have insignificant impact on knowledge dynamics, while unreliable communication channels undermine seriously and persistently the process of knowledge acquisition [8]. A comparison of four different selection rules (\$) (CF7) has been investigated, revealing that knowledge attainment can be significantly improved, simply by raising the local awareness of agents, in order to be able to implement more fruitful selections among their neighboring agents [9]. Concerning the issue of "filtering" agents ( $\mathbb{f}$ ) who are considered inappropriate for knowledge acquisition (CF8), both knowledge gain and knowledge stock become higher when the adopted "knowledge-exchange threshold" is larger [20]. Concerning the impact of the order of implementation of "Selection" versus "Filtering" (CF9), it was found [9] that knowledge attainment is significantly faster, if the conventional $[13,14,18,20]$ rule of "Selection" before "Filtering" ( $\mathbb{E} \Phi)$ is reversed: "Selection" after "Filtering" (\$fI). Concerning attacks on knowledge networks (CF10), it was found [24] that the damage caused by removing nodes is ordered, namely, \{high degree\} > $\{$ high knowledge $\}>\{$ random $\}>\{$ low knowledge $\}$, where $\{X\}$ denotes the damage resulting from removing nodes with property $X$. The study of targeted link attacks revealed that "infecting" links is more harmful than "breaking" links [8]. Concerning (CF11), it was found that, if agents implement intelligent selections (first "filter out" the in-neighbors of lower knowledge and then "select"), then knowledge spread is much faster compared to placing experts in key network positions [10].

The conventional agent-based models for knowledge diffusion in networks are limited to random mindless interactions among the agents $[13,14,18,20]$. However, in real communication networks, the agents (human beings or intelligent machines) are able to make mindful decisions for knowledge acquisition. This is the key distinction of intelligent interacting agents compared to mindless colliding molecules, involved in the usual diffusion mechanism resulting from accidental collisions. The decisions of intelligent rational agents are realized under bounded rationality [25,26]. Agents with higher intelligence have lower bound of rationality. Moreover, the conventional agent-based models for knowledge diffusion in networks assume implicitly that diffusion takes place on "frozen" (static) networks [12-18,23,24,27,28]. As a result, evolution is restricted to the evolution of agents' knowledge only. In reality, however, the network is also evolving, as the weights change, due to the interplay between knowledge change and structural change. As knowledge changes, the weights of the links adapt accordingly (learning), and conversely, as the weights change, the resulting knowledge transfers are enhanced or limited. Open-SourceSoftware developer networks and scientific collaboration networks are well-known realworld examples, where the co-evolution of links' weights with agents' knowledge takes place [20]. We also investigated network co-evolution in 4 real organizational networks, where we found that structural balance emerges concurrently with attitude polarization or domination [29].

The goal of this work is to model and explore the diffusion of knowledge in co-evolving networks of intelligent interacting agents. Using agent-based modeling, the knowledge is diffused due to local microscopic interactions among the agents. We shall formulate the co-evolution of knowledge and weights at the microscopic level of "agent-to-agent" interaction.

We investigate the impact of two conditioning factors, namely, (CF7) and (CF3), on the emerging dynamics of network co-evolution. The impact of the selection rule (\$) (CF7) is assessed in terms of different probabilistic selection-decision rules. These rules correspond to different bounds of rationality and therefore to different intelligence levels of the selecting agents. Higher intelligence is associated with lower bound of rationality. The impact of innovative agents (CF3), is assessed in terms of different innovation rates and different number of "top innovators". 
The paper is organized as follows. The relevant definitions and assumptions of our model are presented in Section 2. The parameter values of the simulations, as well as the assessment criteria for the emerging dynamics are presented in Section 3. The impact of different probabilistic selection rules (CF7), of different innovation rates (CF3), of different numbers of "top-innovators" (CF3) and of different network sizes in Sections 4-7 correspondingly. The key findings of our work are summarized and discussed in Section 8.

\section{The Model}

We formulate knowledge diffusion in networks of agents, assuming that each agent selects another agent to gain knowledge, at each time $t=0,1, \ldots$. We provide below the relevant definitions and modeling assumptions.

\section{Definition 1. (Knowledge Networks).}

Knowledge networks consist of agents, denoted as $\kappa=1,2, \ldots, N$, with knowledge levels $\psi_{K}(t)$ at time $t=0,1, \ldots$ Knowledge levels are non-negative real values $\psi_{\kappa}(t) \geq 0$ so that, agents with higher knowledge level are effectively more knowledgeable than agents with lower knowledge level. Knowledge levels may be assessed by experts using any methodology [30]. The links of the network are the communication channels between the agents through which knowledge is transferred. Each link $\lambda \rightarrow \kappa$ is characterized by the corresponding weight $w_{\lambda \rightarrow \kappa}(t)=w_{\lambda \kappa}(t)$. The weight $w_{\lambda \kappa}(t)$ incorporates the knowledge transfer efficiency from agent $\lambda$ to agent $\kappa$, taking values $0 \leq w_{\lambda \kappa}(t) \leq 1$. The diagonal weight (self-weight) $w_{\kappa \kappa}(t)$ incorporates the capability of agent $\kappa$ for innovation, taking non-negative values $w_{\kappa \kappa}(t) \geq 0$.

\section{Assumption 1. (One Agent is Selected at a Time).}

The knowledge upgrade of each agent $\kappa$ during the time interval $(t, t+1]$ is the difference: $\psi_{\kappa}(t+1)-\psi_{\kappa}(t)$. The durations $(t, t+1]$ are selected-specified so that the knowledge of the agents may change by one "link event" only among their in-neighbors. In this way, the reduction of efficiency and reliability associated with multi-tasking is avoided [31-33]. However, in the other direction, an agent may have more than one disciples at the same time [12,17,34].

\section{Definition 2. (Probabilistic Selection Rules for Knowledge Acquisition).}

The selection of the in-neighbor $\lambda$ by agent $\kappa$, with $\lambda \neq \kappa$, for knowledge acquisition is specified by the value of the selection-decision function $\mathfrak{D}_{K}^{\mathbb{S}}(t)$. Each selection function $\mathfrak{D}_{K}^{\mathbb{S}}(t)$ implements some selection rule $\$$, specified by the corresponding selection probability $p_{\lambda \kappa}^{\$}(t)$. For example, $\mathfrak{D}_{\kappa}^{\Phi}(t)=\lambda$ means that agent $\lambda$ was selected by agent $\kappa$ at time $t$ with probability $p_{\lambda \kappa}^{\Phi}(t)$. Each selection rule $\$$ indicates the bound of rationality of the intelligent, rationally selecting agent $\kappa[25,26]$. Agents with lower bound of rationality are considered as more intelligent. The bound of rationality is lower, when decisions-selections are more effective. We consider four representative probabilistic selection rules [9] (Table 1). The term $\llbracket Q \rrbracket$ is the Iverson bracket [35] which converts Boolean values to numbers 0,1 :

$$
\llbracket Q \rrbracket=\left\{\begin{array}{l}
1, \text { if } Q \text { is True } \\
0, \text { if } Q \text { is False }
\end{array}\right.
$$

\section{Assumption 2. (Knowledge Transfer is Unidirectional).}

Knowledge transfer between two agents may take place only from the agent with higher knowledge to the agent with lower knowledge, i.e., agent $\kappa$ may upgrade his/her knowledge level by the selected in-neighbor agent $\lambda$, only if: $\psi_{\lambda}(t)-\psi_{\kappa}(t)>0$. The opposite direction is never realized. This assumption is a "Filtering Rule" ( $\mathbb{H}$ ) over the set of in-neighbors, regarding the possibility for knowledge upgrade of the selecting agent $\kappa$. The criterion conditioning the "Filtering Rule" $(\mathbb{I})$ is the sign of the knowledge difference $\psi_{\lambda}(t)-\psi_{\kappa}(t)$ between the sender $\lambda$ and the recipient $\kappa$ of knowledge. 
Table 1. Probabilistic selection rules for knowledge acquisition.

\begin{tabular}{|c|c|c|}
\hline Selection Rule $\mathbb{\$}$ & Selection Probability $p_{\lambda \kappa}^{\Phi}(t)$ & Bound of Rationality \\
\hline $\begin{array}{l}\text { Random } \\
\mathbb{S}=r\end{array}$ & $p_{\lambda \kappa}^{\mu}(t)=\frac{\llbracket w_{\lambda \kappa}(t) \neq 0 \rrbracket}{\sum_{v=1}^{N} \llbracket w_{v \kappa}(t) \neq 0 \rrbracket}$ & $\begin{array}{l}\text { All incoming links have equal selection } \\
\text { probability. } \\
\text { There is no rationality, as } \\
\text { communication is random. } \\
\text { The agent } \kappa \text { has no intelligence. }\end{array}$ \\
\hline $\begin{array}{c}\text { Knowledge } \\
\mathbb{S}=k\end{array}$ & 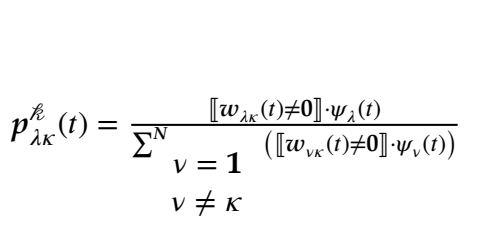 & $\begin{array}{l}\text { More knowledgeable in-neighbors have } \\
\text { higher selection probability } \\
\text { Rationality is limited to the awareness of } \\
\text { the knowledge of the in-neighbors. } \\
\text { The agent } \kappa \text { has knowledge-based } \\
\text { intelligence. }\end{array}$ \\
\hline $\begin{array}{l}\text { Weight } \\
\mathbb{S}=w\end{array}$ & 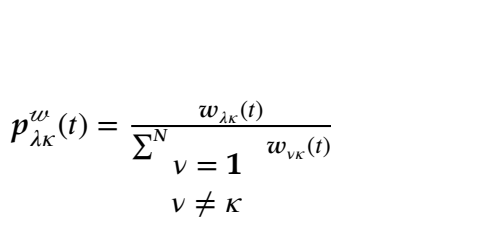 & $\begin{array}{l}\text { Higher incoming weights have higher } \\
\text { selection probability. } \\
\text { Rationality is limited to the awareness of } \\
\text { the incoming weights. } \\
\text { The agent } \kappa \text { has weight-based } \\
\text { intelligence. }\end{array}$ \\
\hline $\begin{array}{c}\text { Knowledge-Weight } \\
\quad \$=w k\end{array}$ & $p_{\lambda \kappa}^{w \kappa}(t)=\frac{w_{\lambda \kappa}(t) \cdot \psi_{\lambda}(t)}{\sum_{\substack{\nu \\
\nu}}\left(w_{v \kappa}(t) \cdot \psi_{v}(t)\right)}$ & $\begin{array}{l}\text { Higher product of weight } \cdot \text { knowled ge } \\
\text { have higher selection probability } \\
\text { Rationality is limited to the awareness of } \\
\text { the incoming weights and the } \\
\text { knowledge of the in-neighbors. } \\
\text { The agent } \kappa \text { has both knowledge and } \\
\text { weight-based intelligence. } \\
\text { The agent } \kappa \text { has finer discrimination } \\
\text { capability, lower bound of rationality } \\
\text { and higher intelligence (Definition } 2) \text {, } \\
\text { compared to the other cases. }\end{array}$ \\
\hline
\end{tabular}

Definition 3. (Knowledge Transfer Dynamics).

The Knowledge Transfer Dynamics for each agent $\kappa$ is as follows:

$$
\psi_{\kappa}(t+1)=\psi_{\kappa}(t)+\sum_{\substack{\nu=1 \\ \nu \neq \kappa}}^{N} \Phi_{\nu \kappa}(t)
$$

where $\Phi_{\nu \kappa}(t)$ is the formula for Knowledge Transfer from agent $\nu$ to agent $\kappa$, with $\nu \neq \kappa$ :

$$
\Phi_{\nu K}(t)=\llbracket \mathfrak{D}_{\kappa}^{\Phi}(t)=v \rrbracket \cdot \llbracket \psi_{\nu}(\boldsymbol{t})-\psi_{\kappa}(t)>0 \rrbracket \cdot w_{\nu K}(t) \cdot\left[\psi_{\nu}(\boldsymbol{t})-\psi_{\kappa}(t)\right]
$$

Remark 1. The Knowledge Transfer Dynamics Equation is stochastic, non-linear and nonhomogeneous.

The Diffusion Equation (1) is:

- $\quad$ Stochastic due to the probabilistic selection $\mathfrak{D}_{K}^{\mathbb{S}}(t)$ (Definition 2, Table 1);

- $\quad$ Non-linear due to the term $\llbracket \psi_{\nu}(t)-\psi_{K}(t)>0 \rrbracket$ (Assumption 2);

- Non-homogeneous due to the different, non-stationary weights $w_{v \kappa}(t)$ (Definition 1).

The Iverson Bracket $\llbracket \mathfrak{D}_{\kappa}^{\Phi}(t)=v \rrbracket$ incorporates the probabilistic selections of the agents for knowledge acquisition into the dynamic law of diffusion. The Iverson Bracket $\llbracket \psi_{\nu}(t)-\psi_{\kappa}(t)>0 \rrbracket$ is serving as a "valve", indicating the allowed direction of knowledge, from the more knowledgeable to the less knowledgeable agent. Knowledge Transfer is proportional to the knowledge difference $\psi_{v}(t)-\psi_{\kappa}(t)$ between the provider $v$ and the recipient $\kappa$. This assumption is common in knowledge transfer models, as for example, Formula (2) in [12], Formula (3) in [13], Formula (6) in [14], For- 
mula (2) in [17], Formula (1) and (3) in [18], Formula (3) in [23], Formula (1) in [27], Formula (4) in [24], Formula (3) and (4) in [36], Formula (3) in [21] and Formula (1) in [28]. However, the diffusion Equation (1) is a generalization of the above models in order to have the possibility to implement different selection rules $\$$ (Table 1), corresponding to intelligent, non-random interactions at the local-microscopic level. The analogous equation for attitude-opinion change incorporates the implementation of selection rules for communication and social influence [37].

\section{Definition 4. (Evolution of the Off-Diagonal Weights).}

We define a local learning rule for the evolution of the off-diagonal weights $w_{\lambda \kappa}(t)$, which is driven by the selections $\mathfrak{D}_{\kappa}^{\$}(t)$ of the individual agents as follows:

- If the in-neighbor agent $\lambda$ is selected $\left(\mathfrak{D}_{\kappa}^{\$}(t)=\lambda\right)$, the corresponding weight $w_{\lambda \kappa}(t)$ may change due to the relevance feedback learning.

- If the in-neighbor agent $\lambda$ is not selected $\left(\mathfrak{D}_{K}^{\Phi}(t) \neq \lambda\right)$, the corresponding weight $w_{\lambda \kappa}(t)$ is subjected to decay with time $t$.

In this first exploratory work, we shall assume the simplest case of linear decay, with decay parameter $\zeta$, taking values $0<\zeta<1$. The parameter $\zeta$ corresponds to the speed of weakening of the communication channels, resulting from the passage of time. A lower value of decay parameter $\zeta$ makes the weight $w_{\lambda \kappa}(t)$ vanish more quickly. We assume for simplicity the same decay parameter $\zeta=0.99$ for all agents $\kappa=1,2, \ldots, N$. In other words, each weight $w_{\lambda \kappa}(t)$ is weakening by $1 \%$ for each time $t$ not selected. The evolution formula for the off-diagonal weights $w_{\lambda \kappa}(t)$ is as follows:

$$
w_{\lambda K}(t+1)=\llbracket \mathfrak{D}_{K}^{\mathbb{S}}(t)=\lambda \rrbracket \cdot\left[\left(1-L_{\lambda K}(t)\right) \cdot w_{\lambda K}(t)+L_{\lambda K}(t) \cdot F_{\lambda K}(t)\right]+\llbracket \mathfrak{D}_{K}^{\mathbb{S}}(t) \neq \lambda \rrbracket \cdot w_{\lambda K}(t) \cdot \zeta
$$

$F_{\lambda \kappa}(t)=\llbracket \psi_{\lambda}(t)-\psi_{\kappa}(t)>0 \rrbracket$ is the Relevance Feedback, which in this first exploratory work takes binary values, 0 or 1 , depending on whether some knowledge is transferred via the channel $\lambda \rightarrow \kappa$. If some knowledge is transferred, the channel efficiency (weight) is enhanced, otherwise it weakens.

$\mathbf{L}_{\lambda \kappa}(t)=\ell \cdot \llbracket \boldsymbol{F}_{\lambda \kappa}(t)=\mathbf{1} \rrbracket+(\mathbf{1}-\ell) \cdot \llbracket \boldsymbol{F}_{\lambda \kappa}(t)=\mathbf{0} \rrbracket$ is the Learning Function, where $\ell$ is $a$ parameter implying the learning rate of the weights, taking values $0<\ell<0.5$. The learning function $L_{\lambda \kappa}(t)$ takes values $\ell$ or $1-\ell$, distinguished according to the value of the relevance feedback $\boldsymbol{F}_{\lambda \kappa}(t)$. That is because we want to incorporate a "slow-positive" $\left(\boldsymbol{F}_{\lambda \kappa}(t)=1\right)$ and "fast-negative" $\left(\boldsymbol{F}_{\lambda \kappa}(t)=0\right)$ effect of the relevance feedback $\boldsymbol{F}_{\lambda \kappa}(t)$ on the evolutionary dynamics of the weights. If the relevance feedback is positive $\left(\boldsymbol{F}_{\lambda \kappa}(t)=1\right)$, then learning $L_{\lambda_{K}}(t)$ is low (below 0.5), equal to $\ell \in(0,0.5)$. On the contrary, if the relevance feedback is zero $\left(\boldsymbol{F}_{\lambda \kappa}(t)=0\right)$, then learning $L_{\lambda \kappa}(t)$ is high (above 0.5), equal to $1-\ell \in(0.5,1)$. In this first exploratory work, we shall assume learning rate $\ell=0.4$.

\section{Assumption 3. (Periodic Innovation).}

All agents create new knowledge $\left(w_{\kappa \kappa}(t)>0\right.$ for $\kappa=1,2, \ldots, N$, Definition 1) periodically with period $T$. The period $T$ is the innovation rate of the system.

Definition 5. (Selection Function for Innovation Production and Knowledge Acquisition).

Taking into account Assumption 3, the selection function $\mathfrak{D}_{K}(t)$ incorporates: $(a)$ the selection of distinct agents $v($ with $v \neq \kappa)$ for knowledge acquisition, based on some selection rule $\mathbb{S}$ $\left(\mathfrak{D}_{\kappa}(t)=\mathfrak{D}_{\kappa}^{\mathbb{S}}(t)=v\right)$, as well as, $(b)$ the self-selection of agent $\kappa\left(\mathfrak{D}_{\kappa}(t)=\kappa\right)$ for innovation production with period $T$. Therefore, the selection function $\mathfrak{D}_{K}(t)$ is defined as follows:

$$
\mathfrak{D}_{\kappa}(t)=\llbracket \frac{t}{T}=\left\lfloor\frac{t}{T}\right\rfloor \rrbracket \cdot \kappa+\llbracket \frac{t}{T} \neq\left\lfloor\frac{t}{T}\right\rfloor \rrbracket \cdot \mathfrak{D}_{K}^{\mathbb{S}}(t)
$$

where $x$ is the floor function which returns the largest integer not greater than $x$. For example, $2.2=2$ and $2.9=2$. 
Definition 6. (Knowledge Dynamics Equation).

The Knowledge Dynamics Equation for each agent $\kappa$ is as follows:

$$
\psi_{\kappa}(t+1)=\psi_{\kappa}(t)+\sum_{\nu=1}^{N} \Phi_{\nu \kappa}(t)
$$

The off-diagonal elements $\Phi_{v K}(t)$ correspond to Knowledge Transfer from each agent $v$ to agent $\kappa$, with $v \neq \kappa$, (Definition 3$)$. The diagonal element $\Phi_{\kappa \kappa}(t)$ corresponds to Innovation Production from agent $\kappa$ (see Definition below). Knowledge dynamics in networks is understood and modeled as spread of knowledge, due to discrete diffusion in the network. This diffusion process is in general non-uniform and non-isotropic, as diffusion in disordered systems [38] and anomalous biased diffusion [39]. Our model allows moreover the simulation of "active" interactions among the agents, introduced by selections-decisions characterized by awareness, resulting in higher intelligence. These "intelligent selections" (Definition 2, Table 1) are mechanisms of "active" diffusion, interpreted as awareness propagation in communication networks [40].

\section{Definition 7. (Innovation Production Dynamics)}

The Innovation Production Dynamics for each agent $\kappa$ is as follows:

$$
\psi_{\kappa}(t+1)=\psi_{\kappa}(t)+\Phi_{\kappa \kappa}(t)
$$

where $\Phi_{\kappa \kappa}(t)$ is the formula for Innovation Production from agent $\kappa$ :

$$
\Phi_{\kappa \kappa}(t)=\llbracket \mathfrak{D}_{\kappa}(t)=\kappa \rrbracket \cdot w_{\kappa \kappa}(t) \cdot \psi_{\kappa}(t)
$$

Remark 2. Innovation production is proportional to knowledge level $\psi_{\kappa}(t)$.

This assumption is common in innovation production models, as for example, formula (1) in [12], formula (1) in [34], formula (1) in [14], Formula (1) in [17], Formula (5) in [23], Formula (2) in [28]. We want to focus on the impact of different innovation rates $T$ (Assumption 3). Therefore, in this first exploratory work, we shall assume stationary self-weights $\left(w_{\kappa \kappa}(t)=w_{\kappa \kappa}\right)$ with values discussed in Section 3.

Remark 3. Weights-Knowledge co-evolution via local "agent-to-agent" interactions.

The co-evolution of weights and knowledge is formulated in terms of evolution equations for weights (2) and knowledge (4). This Weights-Knowledge co-evolution equation is a non-linear stochastic difference equation, incorporating the actual interplay of weights with knowledge levels in time. The interdependence of weights with knowledge levels is formulated at the local microscopic level of "agent-to-agent" interaction, the result of which is twofold (Figure 1): On one hand, there is an upgrade of agents' knowledge due to the diffusion process. On the other hand, at the same time, there is a mutually dependent update of agents' weights due to the "experience" of communication (feedback) with the selected agent. The simultaneous update of knowledge and weights is the key distinction of our model, compared to other agent-based models for knowledge network co-evolution, where the updates of knowledge and weights, are not realized simultaneously. At each time step, they propose a random choice for: either link rewiring only, or knowledge diffusion only [20,21], excluding simultaneous update. Moreover, in the existing literature of agent-based models for knowledge diffusion in networks $[18,20,21,41]$, the evolution of weights from $w_{\lambda \kappa}(t)$ to $w_{\lambda K}(t+1)$ is imposed exogenously. Thus, the evolution of weights and knowledge is not a mutually dependent evolution. On the contrary, in our model, we observe from equation (2) that the weights evolve to the value $w_{\lambda \kappa}(t+1)$ depending on the previous values of both weights $w_{\lambda \kappa}(t)$ and knowledge levels $\psi_{\kappa}(t)$ :

$$
w_{\lambda K}(t+1)=f\left(w_{\lambda K}(t), \mathfrak{D}_{\kappa}^{\mathbb{S}}\left(w_{\lambda K}(t), \psi_{\lambda}(t)\right), \boldsymbol{F}_{\lambda K}\left(\psi_{\lambda}(t), \psi_{K}(t)\right)\right)
$$




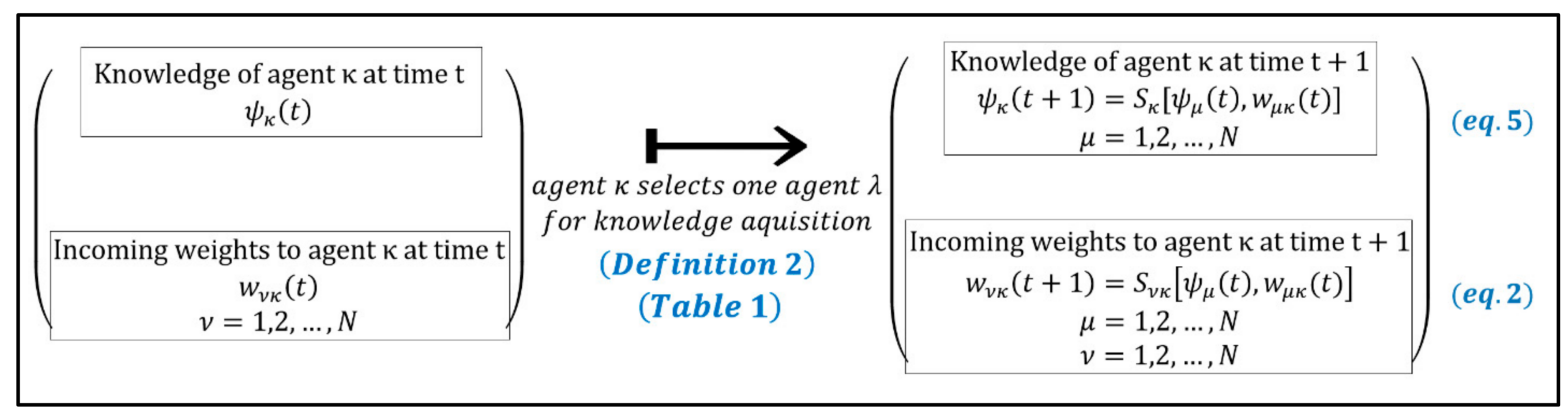

Figure 1. Local Mechanism for Interdependent Co-evolution of weights and knowledge for each agent $\kappa, \kappa=1,2, \ldots, N$, at each time $t=0,1,2, \ldots$ Knowledge and weights co-evolve, because the change of knowledge and weights depends on the values of both knowledge and weights at the previous step. This interdependence of knowledge and weights is conditioning the network evolution at each step.

This is a learning mechanism [42] where co-evolution is intrinsic/endogenous.

\section{Estimating the Dynamics of Network Co-Evolution}

In order to focus on the impact of factors (CF7) and (CF3) on the emerging coevolutionary dynamics, we study fully connected networks [43]. The impact of the selection rule ( $\$$ ) (CF7) is assessed in terms of 4 different probabilistic selection rules (Definition 2, Table 1). The impact of the innovative agents (CF3), is assessed in terms of 3 different innovation rates (Assumption 3 ) and 3 different number of "top innovators". We also examine the dependence on network size. The initial network setting is presented in Table 2 and the values of parameters in the simulations in Table 3.

Table 2. Initial network setting.

\begin{tabular}{cl}
\hline Knowledge & All agents have Equal Initial knowledge level, with value $\psi_{\kappa}(t)=\mathbf{1}$ \\
\hline & $\begin{array}{l}\text { Off-diagonal weights are initially equal, with value } w_{\lambda \kappa}(t)=\mathbf{0 . 5} \\
\text { Fully connected network structure with evolving weights }\end{array}$ \\
\cline { 2 - 2 } Links & $\begin{array}{l}\text { Diagonal weights are constant in time (Remark } 2) \\
\text { The value of most diagonal weights is } w_{\kappa \kappa}=0.05 .\end{array}$ \\
& We assume a small number $(5 \%, 10 \%, 15 \%)$ of randomly distributed \\
& "top innovators" (highly innovative agents) with $w_{\kappa \kappa}=\mathbf{0 . 5}$ \\
\hline
\end{tabular}

Table 3. Simulations.

\begin{tabular}{|c|c|}
\hline Parameter & Values \\
\hline $\begin{array}{l}\text { Selection Rule s (CF7) } \\
\text { for knowledge acquisition } \\
\text { (Definition 2, Table 1) }\end{array}$ & $\begin{array}{l}\text { Random } \Phi=r \\
\text { Knowledge } \Phi=k \\
\text { Weight } \Phi=w \\
\text { Knowledge and Weight } \Phi=w k\end{array}$ \\
\hline $\begin{array}{c}\text { Innovation Rate (CF3) } \\
\text { (period } T \text { for innovation production, Assumption 3) }\end{array}$ & $\begin{array}{l}\text { Low } T=15 \text { steps } \\
\text { Medium } T=10 \text { steps } \\
\text { High } T=5 \text { steps }\end{array}$ \\
\hline $\begin{array}{l}\text { Number of Top Innovators (CF3) } \\
\text { (highly innovative agents, Table 2) }\end{array}$ & $\begin{array}{l}\text { Few } 5 \% \\
\text { Some } 10 \% \\
\text { Many } 15 \%\end{array}$ \\
\hline Network Size & $\begin{array}{l}\text { Small } N=100 \text { agents } \\
\text { Medium } N=200 \text { agents } \\
\text { High } N=300 \text { agents }\end{array}$ \\
\hline
\end{tabular}

The off-diagonal weights $w_{\lambda \kappa}$, incorporating the knowledge transfer efficiency from agent $\lambda$ to agent $\kappa$, take values within the interval $[0,1]$. We assume that the initial value is 
at the center: $w_{\lambda k}=0.5$. The selected values in simulations are context-dependent, for example, (a) in simulations of collective invention dynamics [12], knowledge diffusion [13] and knowledge transfer $[17,28]$, the off-diagonal weights $w_{\lambda \kappa}$ take values within the same interval $[0,1],(b)$ in simulations of the dependence of knowledge transfer on the connection structure, the off-diagonal weights $w_{\lambda \kappa}$ take values within the interval $[0,0.5]$ [18].

Concerning network size, it is not expected that simulations with more than 300 agents will add significantly to the results. This is confirmed by several works on knowledge dynamics in networks, namely, (a) simulations with 150 agents for tacit knowledge transmission in online social networks [44] "managers must make sure the number of employees in an organization is less than 150 if the organization means to run efficiently and becomes the incubator of the propagation of knowledge and thoughts. 150 is a tipping point. If the number of employees is more than 150, many organizations will break up" [45,46], (b) simulations with 100 agents for knowledge diffusion performance in R\&D collaboration networks [14], (c) simulations with 200 agents for coevolution of endogenous knowledge networks and knowledge creation [47], (d) simulations with 200 agents for knowledge diffusion dynamics with faceto-face interactions [48], (e) simulations with 100 agents for knowledge diffusion in social networks, manifesting serendipity [49].

The emerging dynamics of network co-evolution is assessed in terms of the following:

- The average knowledge of the agents and the "knowledge inequality" (standard deviation of knowledge of the agents);

- The average selection entropy of the agents;

- The out-degree centralization of the network.

The definition for the average knowledge of the agents is as follows:

Definition 8. (Average Knowledge).

$$
\bar{\psi}(\boldsymbol{t})=\frac{\sum_{\kappa=1}^{N} \psi_{\kappa}(t)}{N}
$$

The "knowledge inequality" of the agents may be estimated by the standard deviation $[12-14,17,23,27,36]$ :

Definition 9. ("Knowledge Inequality"-Standard Deviation of Knowledge).

$$
\sigma_{\psi}(t)=\sqrt{\frac{\sum_{\kappa=1}^{N}\left[\psi_{\kappa}(t)-\bar{\psi}(t)\right]^{2}}{N}}
$$

The relevant definitions for the average selection entropy of the agents are:

Definition 10. (Selection Entropy).

The selection entropy $\mathcal{S}_{\kappa}(t)=\mathcal{S}_{\kappa}^{\Phi}(t)$ of agent $\kappa$ is the "in-entropy" of node $\kappa$, computed from the selection probabilities $\boldsymbol{p}_{\lambda K}^{\mathbb{S}}(t)$ of Table 1 as follows:

$$
\mathcal{S}_{\kappa}(t)=\mathcal{S}_{\kappa}^{\Phi}(t)=-\sum_{\substack{\lambda=1 \\ \lambda \neq \kappa}}^{N} p_{\lambda \kappa}^{\Phi}(t) \cdot \log _{2}\left[p_{\lambda \kappa}^{\Phi}(t)\right]
$$

The normalized selection entropy $\mathscr{I}_{\kappa}(t)=\mathscr{I}_{\kappa}^{\$}(t)$ of agent $\kappa$ is as follows:

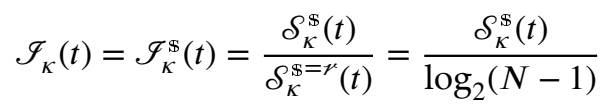


The average normalized selection entropy $\overline{\mathscr{I}}(t)=\overline{\mathcal{I}^{\Phi}}(t)$ of the agents of the network is as follows:

$$
\overline{\mathscr{I}}(t)=\overline{\mathscr{I}^{\Phi}}(t)=\frac{\sum_{\kappa=1}^{N} \mathscr{I}_{\kappa}^{\Phi}(t)}{N}
$$

We shall use the average normalized selection entropy $\overline{\mathscr{I}}(t)$ to estimate the "Selection Entropy".

The relevant definitions for the centralization of the network are:

Definition 11. (Weighted Out-Degree).

The weighted out-degree degout $(t)$ of agent $\kappa$ is defined as the sum of the outgoing weights $w_{\kappa \nu}(t)$ to all its first distinct neighbors:

$$
\operatorname{deg}_{\kappa}^{\text {out }}(t)=\sum_{\substack{\nu=1 \\ v \neq \kappa}}^{N} w_{\kappa v}(t)
$$

\section{Definition 12. (Weighted Out-Degree Centrality).}

The weighted out-degree centrality $D E G_{K}^{\text {out }}(t)$ of agent $\kappa$ is defined [50] as the normalized weighted out-degree $\operatorname{deg}_{\kappa}^{\text {out }}(t)$ (Definition 11):

$$
D E G_{\kappa}^{\text {out }}(t)=\frac{\operatorname{deg}_{\kappa}^{\text {out }}(t)}{N-1}
$$

Definition 13. (Weighted Out-Degree Centralization).

The centralization of a network [50,51], for the weighted out-degree centrality $D E G_{\kappa}^{\text {out }}(t)$ (Definition 12), is the fraction:

$$
D E G^{\text {out }}(t)=\frac{\sum_{\kappa=1}^{N}\left[D E G_{\xi}^{\text {out }}(t)-D E G_{\kappa}^{\text {out }}(t)\right]}{N-1}
$$

where: $D E G_{\xi}^{\text {out }}(t)=\max _{\kappa=1,2, \ldots, N}\left\{D E G_{\kappa}^{\text {out }}(t)\right\}$.

The numerator is the sum of differences between the degree centralities of the most central node $\xi$ and all other nodes $\kappa$. The denominator is the theoretically largest sum of such differences in any network of the same size. The theoretically largest sum of such differences is achieved for the directed "star" network topology, where the network consists of one "star" (central) node $\xi$ having maximal out-going weights to all other "satellite" nodes, and "satellite" nodes have no out-going weights. We shall use the weighted out-degree centralization $D E G^{\text {out }}(t)$ to estimate the "Centralization".

\section{The Impact of Intelligence-Selection Rule}

The impact of intelligence of the agents (CF7) (Definition 2, Table 1) on the emerging dynamics of network co-evolution is assessed by performing simulations for the 4 different probabilistic selection rules (Table 3), namely, (1) selection by randomness $(\$=\gamma)$, (2) selection by knowledge level $(s=\hbar)$, (3) selection by link weight $(s=w)$, (4) selection by knowledge level and link weight $(\mathrm{s}=w k)$. Results are illustrated in Figure 2. 

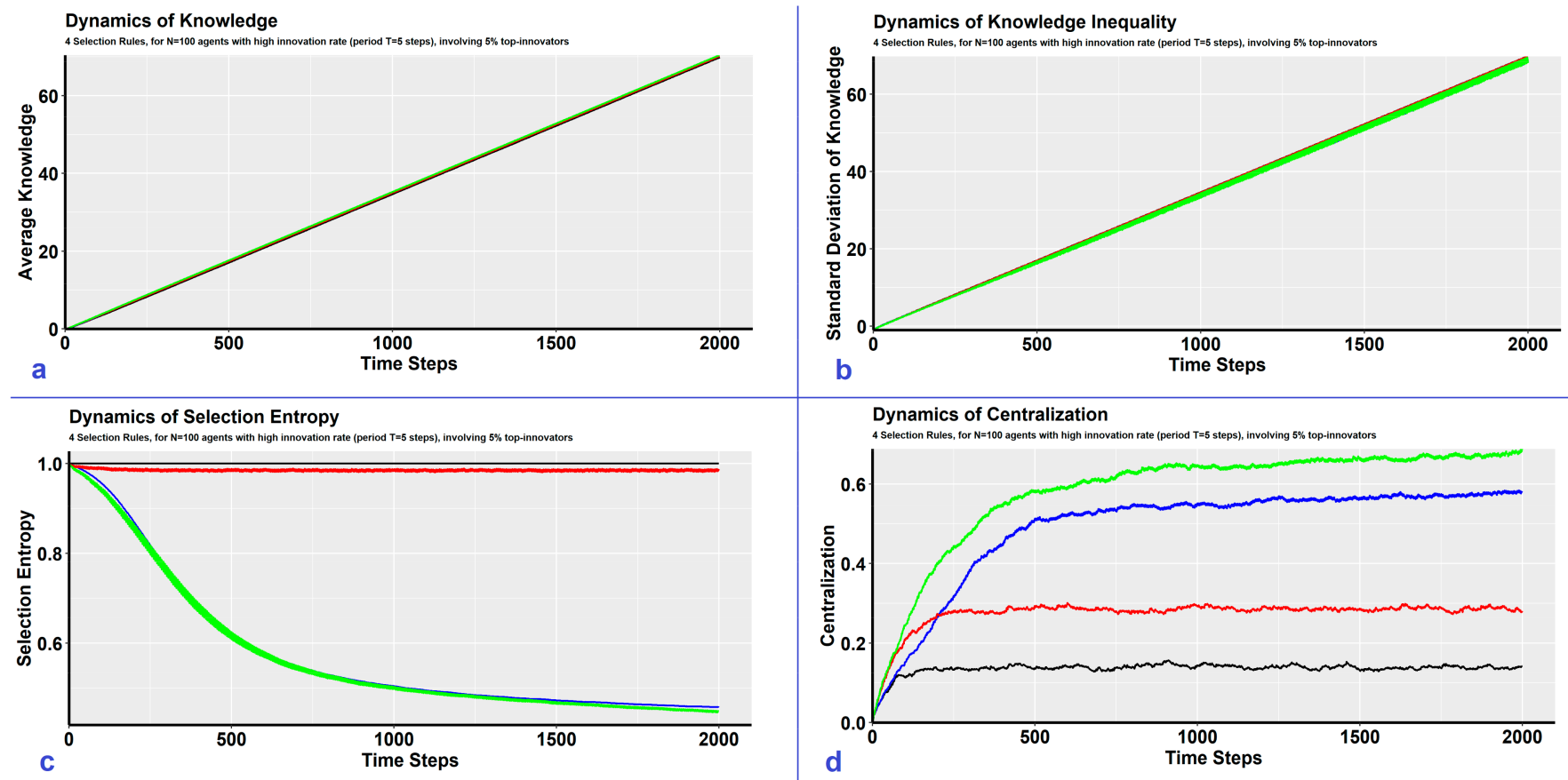

Figure 2. Simulations for the 4 different probabilistic selection rules: (1) random $\$=r$ (black), (2) knowledge $\$=k$ (red), (3) weight $s=w$ (blue), (4) knowledge-weight $\$=w k$ (green). Results are illustrated for: (a) the average knowledge (Definition 8, up left), (b) the standard deviation of knowledge (Definition 9, up right), (c) the selection entropy (Definition 10, down left), (d) the centralization (Definition 13, down right). The y axis for the average knowledge (a, up left) and the standard deviation of knowledge (b, up right) is in log-10 scale, indicating the exponential increase of knowledge dynamics. Results are illustrated for $N=100$ agents with high innovation rate (period $T=5$ steps), involving $5 \%$ top-innovators.

\section{Remark 4. Average knowledge and "knowledge inequality" are both increasing exponen- tially in time.}

We observe in Figure 2a,b that the average knowledge, as well as the "knowledge inequality" (standard deviation of knowledge), are both rising exponentially in time (y axis is in log-10 scale), with more or less the same rate $\simeq 3.5 \%$. The intelligence (Definition 2, Table 1) of communicating agents has negligible impact on the emerging dynamics of average knowledge, as well as on knowledge inequality, as the diagrams coincide.

\section{Remark 5. Intelligent agents reduce selection entropy.}

We observe in Figure $2 c$ that among intelligent interacting agents, the selection entropy is decreasing in time. The selection entropy of non-intelligent agents, communicating randomly $(\mathrm{s}=r$, black line in Figure 2c), does not decrease. This follows from the uniform selection probability (Table 1). The decrease is negligible in the case of knowledge aware intelligent agents ( $\$=\kappa$, red line in Figure 2c). This follows from the fact that the selection probability for knowledge aware intelligent agents is very close the uniform selection probability, because almost all neighbors have more or less the same knowledge, except the few top innovators (Table 2). On the contrary, the selection entropy of weight aware intelligent agents $(s=w$, blue line in Figure $2 c)$ and of knowledge and weight aware intelligent agents ( $\mathrm{s}=$ wk, green line in Figure $2 c$ ) is rapidly decreasing almost $50 \%$. The decrease of selection entropy is significant when intelligent agents are aware of the weights, because the selection probability diverges rapidly from the uniform distribution, due to the evolution formula for the off-diagonal weights $w_{\lambda \kappa}(t)$ (Definition 4). More specifically, the initial selection probability (Table 1) for a link is very low, due to the fully connected structure and the initial equal weights (Table 2). In the next step, the weights of non-selected links are weakened with decay parameter $\zeta$, while the weights of selected links may either increase or decrease, depending on the knowledge transfer realized (Definition 4). The resulting selection probability is rapidly diverging from the initial uniform distribution. 


\section{Remark 6. Intelligent agents centralize the network.}

The increase of network centralization is clearly observed in Figure $2 d$. However, the increase depends significantly on the intelligence of the agents. Centralization increases about $13 \%$ for non-intelligent agents, communicating randomly ( $\$=r$, black line in Figure $2 d)$. This increase results from the evolution formula for the off-diagonal weights $w_{\lambda \kappa}(t)$ (Definition 4). At each time, most of the links are not selected, and therefore, centralization increases. As intelligent agents select non-randomly, we observe for the same reason the emergence of stronger centralizations, namely, about $27 \%$ for knowledge aware intelligent agents ( $\mathrm{s}=\hbar$, red line in Figure $2 c$ ), about $58 \%$ for weight aware intelligent agents $(\mathrm{s}=w$, blue line in Figure $2 d$ ) and about $70 \%$ for weight and knowledge aware intelligent agents ( $\$=w h$, green line in Figure $2 d$ ).

\section{The Impact of the Innovation Rate}

The impact of the innovation rate of the agents (CF3) (Assumption 3) on the emerging dynamics of network co-evolution is assessed by performing simulations for the 3 different innovation rates (Table 3), namely, (1) low innovation rate with period $T=15$ steps, (2) medium innovation rate with period $T=10$ steps, (3) high innovation rate with period $T=5$ steps. Results are illustrated in Figure 3.
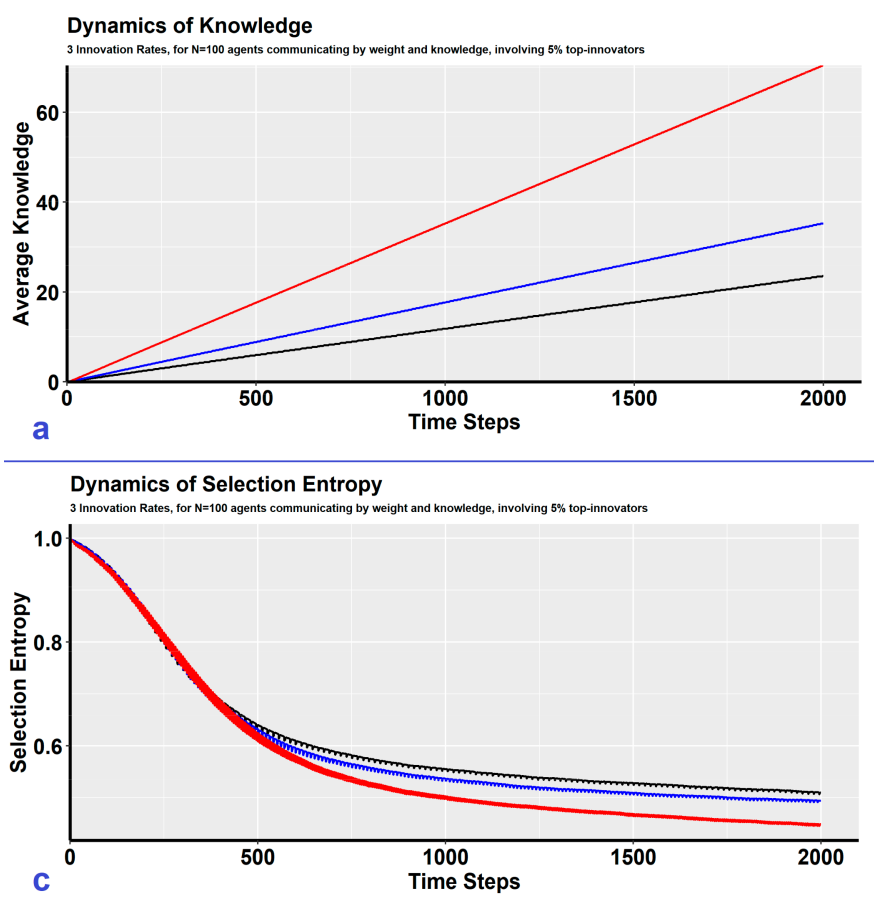
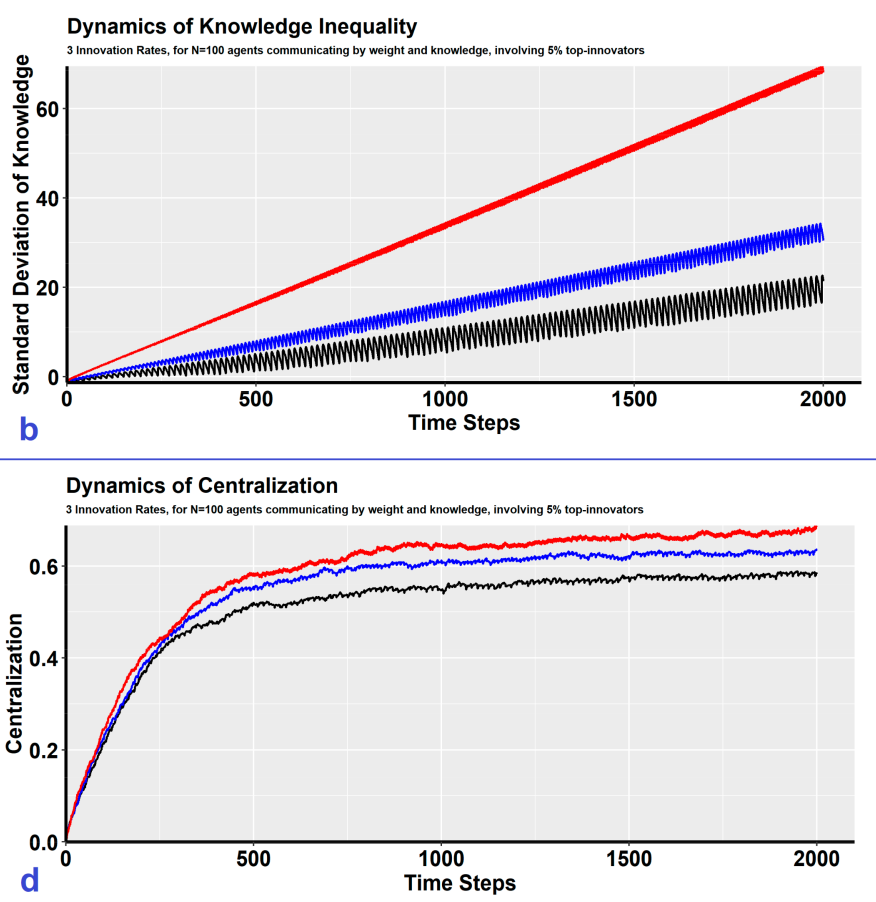

Figure 3. Simulations for the 3 different innovation rates: (1) low rate with period $T=15$ steps (black), (2) medium rate with period $T=10$ steps (blue), (3) high rate with period $T=5$ steps (red). Results are illustrated for: (a) the average knowledge (Definition 8, up left), (b) the standard deviation of knowledge (Definition 9, up right), (c) the selection entropy (Definition 10, down left), (d) the centralization (Definition 13, down right). The y axis for the average knowledge (a, up left) and the standard deviation of knowledge (b, up right) is in log-10 scale, indicating the exponential increase of knowledge dynamics. Results are illustrated for $N=100$ agents communicating by weight and knowledge $(\$=w k)$, involving $5 \%$ top-innovators.

Remark 7. Higher innovation rates result in higher average knowledge and higher knowledge inequality.

We observe in Figure 3a,b that the average knowledge, as well as the "knowledge inequality" (standard deviation of knowledge), are both rising in time ( $y$ axis is in log-10 scale) exponentially. Higher innovation rates result in higher exponential increase of average knowledge as well as of knowledge inequality. The oscillations of knowledge inequality (Figure 3b) are due to the periodic innovation production (Assumption 3). The oscillations are relatively higher for lower innovation 
rate (black line in Figure 3b). At each instance of innovation, the standard deviation of knowledge is increasing. However, at each step between two successive innovation instances, the standard deviation of knowledge is decreasing, because at these intermediate steps, there is only knowledge diffusion and no innovation.

\section{Remark 8. Higher innovation rates result in stronger selection entropy decrease and stronger network centralization increase.}

The selection entropy (Figure 3c) and the network centralization (Figure 3d) are ranked, with respect to the innovation rate. As the innovation rate increases, both the selection entropy decrease, as well as the network centralization increase, become stronger.

\section{The Impact of the Number of Top Innovators}

The impact of the number of top innovators (CF3) (Assumption 3) on the emerging dynamics of network co-evolution is assessed by performing simulations for the 3 different numbers of top innovators (Table 3), namely, 5\%, 10\%, 15\%. Results are illustrated in Figure 4.
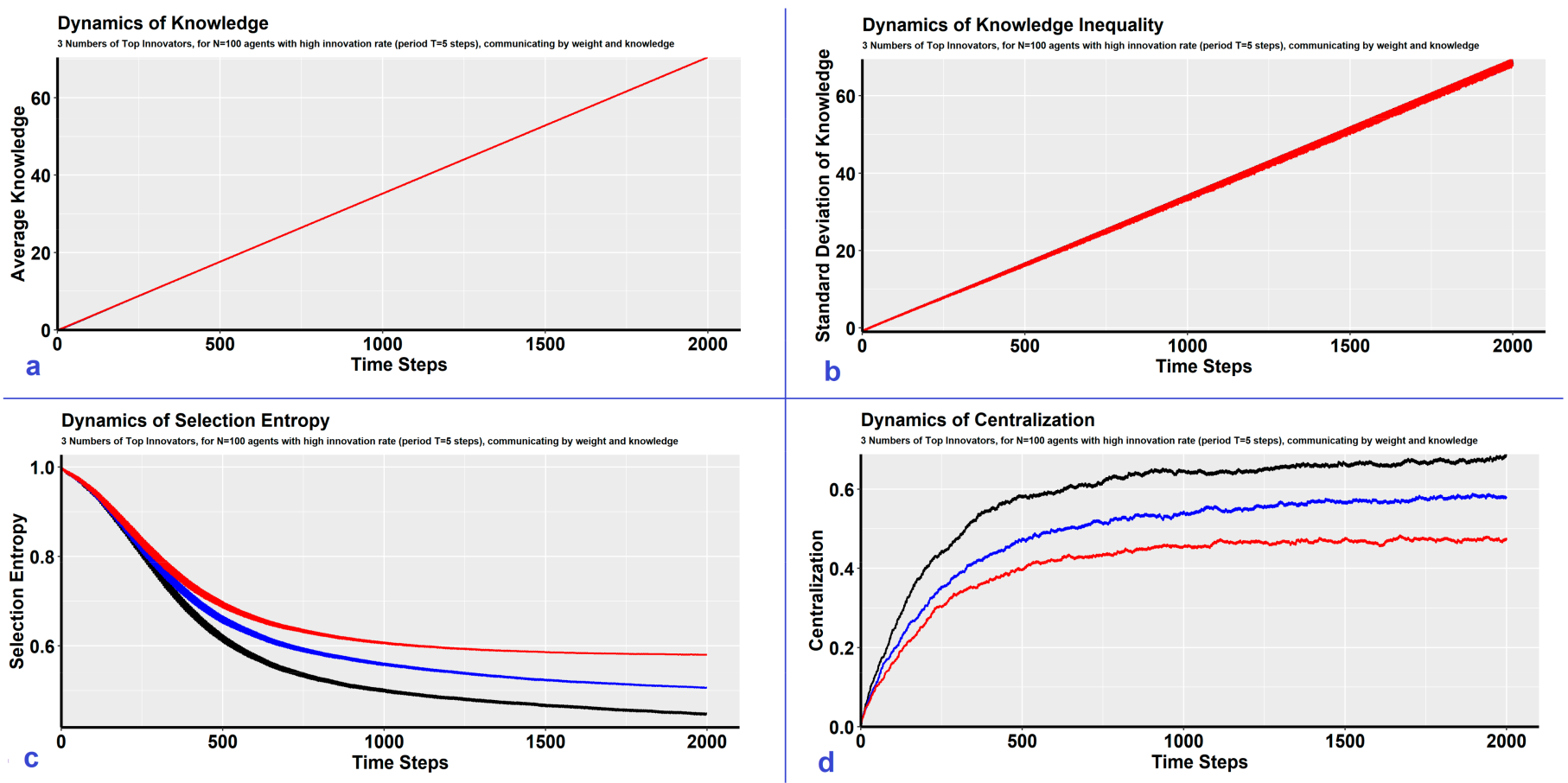

Figure 4. Simulations for the 3 different numbers of top innovators: $5 \%$ (black), $10 \%$ (blue), 15\% (red). Results are illustrated for: (a) the average knowledge (Definition 8, up left), (b) the standard deviation of knowledge (Definition 9, up right), (c) the selection entropy (Definition 10, down left), (d) the centralization (Definition 13, down right). The y axis for the average knowledge (a, up left) and the standard deviation of knowledge (b, up right) is in log-10 scale, indicating the exponential increase of knowledge dynamics. Results are illustrated for $N=100$ agents with high innovation rate (period $T=5$ steps), communicating by weight and knowledge $(\mathbb{s}=w k)$.

Remark 9. Fewer "top-innovators" result in stronger selection entropy decrease and stronger network centralization increase.

We observe in Figure 4a,b that the average knowledge, as well as the "knowledge inequality" (standard deviation of knowledge), are both rising exponentially in time ( $y$ axis is in log-10 scale), with more or less the same rate $\simeq 3.5 \%$. The number of top innovators has negligible impact on the emerging dynamics of average knowledge, as well as on knowledge inequality, as the diagrams coincide. On the contrary, both the selection entropy and the network centralization depend significantly on the number of top innovators. The selection entropy (Figure 4c) and the network centralization (Figure $4 d$ ) are ranked, with respect to the number of top innovators. As the number 
of top innovators decreases, both the selection entropy decrease, as well as the network centralization increase, become stronger.

\section{The Impact of Network Size}

The impact of network size on the emerging dynamics of network co-evolution is assessed by performing simulations for the 3 different network sizes (Table 3), namely, $\mathrm{N}=100$ agents, $\mathrm{N}=200$ agents, $\mathrm{N}=300$ agents. Results are illustrated in Figure 5 .
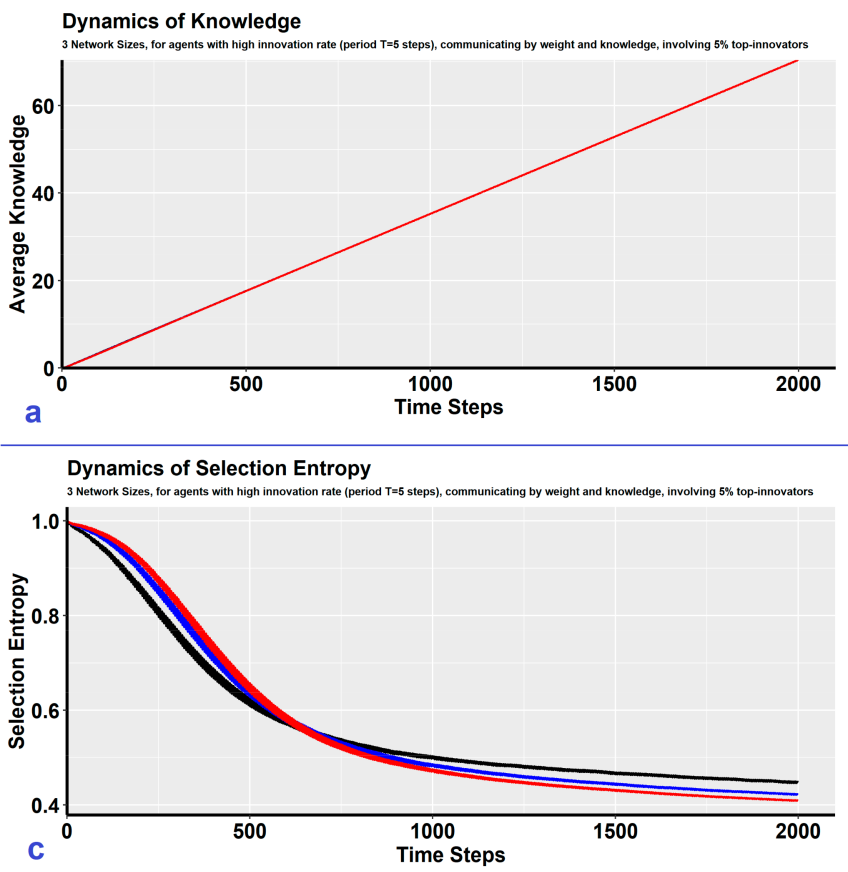
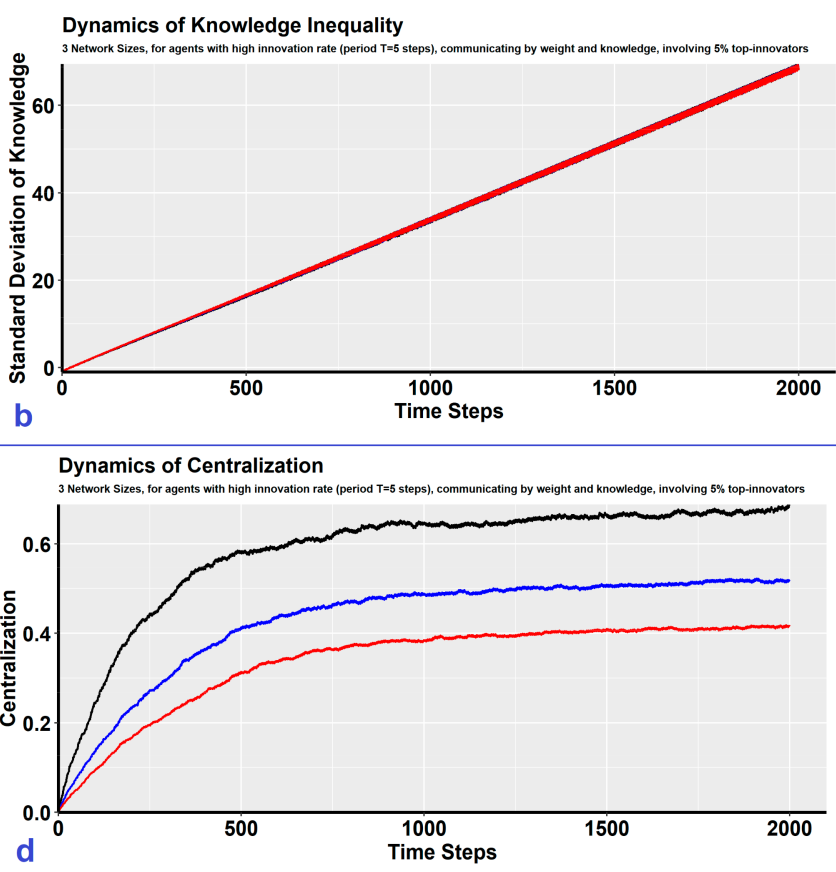

Figure 5. Simulations for the 3 different network sizes: $N=100$ agents (black), $N=200$ agents (blue), $N=300$ agents (red). Results are illustrated for: (a) the average knowledge (Definition 8, up left), (b) the standard deviation of knowledge (Definition 9, up right), (c) the selection entropy (Definition 10, down left), (d) the centralization (Definition 13, down right). The y axis for the average knowledge (a, up left) and the standard deviation of knowledge (b, up right) is in log-10 scale, indicating the exponential increase of knowledge dynamics. Results are illustrated for agents with high innovation rate (period $T=5$ steps), communicating by weight and knowledge $(\$=w k)$, involving $5 \%$ top-innovators.

\section{Remark 10. Smaller networks give rise to stronger centralization increase.}

We observe in Figure 5a,b that the average knowledge, as well as the "knowledge inequality" (standard deviation of knowledge), are both rising exponentially in time ( $y$ axis is in log-10 scale), with more or less the same rate $\simeq 3.5 \%$. The network size has negligible impact on the emerging $d y$ namics of average knowledge as well as on knowledge inequality. Selection entropy depends slightly on network size (Figure 5c). On the contrary, centralization depends significantly on network size (Figure $5 d$ ). For smaller networks, the centralization increase becomes stronger.

\section{Discussion}

The development of "new mathematical formalisms for the co-evolutionary dynamics of states and interactions" has been identified by Thurner, Hanel and Klimek as one of the key directions of future research in the Theory of Complex Systems (Chapter 7 of [52]). San Miguel, Eguíluz and others published on the co-evolution of dynamical states and interactions, addressing the question of network formation [53-58]. In this context, we have modeled and explored the co-evolution of link weights and knowledge levels among intelligent interacting agents. We extended the mathematical models, restricted to random mindless interactions among the agents [13,14,18,20] taking place on a "frozen" (static) network [12-18,23,24,27,28], in two ways: 
- The agents (human beings or intelligent machines) are able to make mindful decisions for knowledge acquisition. The decisions of intelligent rational agents are realized under bounded rationality [25,26]. If the bound of rationality, concerning the selections of other agents for knowledge acquisition is lower, then the agent is considered as more intelligent (Definition 2, Table 1).

- We formulated the co-evolution of the link weights and the knowledge levels at the local microscopic level of "agent-to-agent" interaction. The model describes the "structural plasticity" of the network as a "learning mechanism", where weight updates depend on the previous values of both weights and knowledge levels. As a result, the co-evolution is intrinsic/endogenous (Definition 4, Remark 3).

The goal of this work is to explore the impact of the following:

- The intelligence of the agents, modeled by the selection-decision rule for knowledge acquisition (Definition 2, Table 3) (CF7);

- $\quad$ The innovation rate of the agents (Assumption 3, Table 3) (CF3);

- The number of "top innovators" (Table 3);

- The network size (Table 3).

Our results are summarized in 3 key findings, presented also in Table 4: The network centralization increases as the agents become more intelligent (Remark 6), as the innovation rate increases (Remark 8), as the number of top innovators decreases (Remark 9) and as the network size decreases (Remark 10). The selection entropy decreases as the agents become more intelligent (Remark 5), as the innovation rate increases (Remark 8) and as the number of top innovators decreases (Remark 9). Selection entropy does not depend significantly on the network size (Remark 10). The average knowledge and the knowledge inequality grow exponentially in time (Remarks 4, 7, 9, 10). The rate of exponential growth is increasing as the innovation rate increases. The exponential growth of average knowledge has been observed in several socio-economic systems [59] and scientific knowledge [60]. However, the analysis of exponential knowledge increase for individuals is beyond the scope of this work. Moreover, we observe increasing small oscillations, for lower innovation rates (Remark 7). On the contrary, there is negligible dependence on the intelligence of the agents (Remark 4), on the number of top innovators (Remark 9) and on the network size (Remark 10).

Table 4. Impact of parameters.

\begin{tabular}{|c|c|c|c|c|c|}
\hline & & \multicolumn{4}{|c|}{ Parameter (Table 3) } \\
\hline & & $\begin{array}{c}\text { Increase of } \\
\text { Intelligence } \\
\text { (CF7) }\end{array}$ & $\begin{array}{c}\text { Increase of } \\
\text { Innovation } \\
\text { Rate } \\
\text { (CF3) }\end{array}$ & $\begin{array}{c}\text { Decrease of } \\
\text { Number of } \\
\text { Top } \\
\text { Innovators } \\
\text { (CF3) }\end{array}$ & $\begin{array}{c}\text { Decrease of } \\
\text { Network } \\
\text { Size }\end{array}$ \\
\hline \multirow[t]{3}{*}{$\begin{array}{c}\text { Impact } \\
\text { Assessment }\end{array}$} & $\begin{array}{l}\text { Knowledge } \\
\text { Inequality }\end{array}$ & $\begin{array}{l}\text { negligible } \\
\text { impact } \\
\text { (Figure 2a) } \\
\text { negligible } \\
\text { impact } \\
\text { (Figure 2b) }\end{array}$ & $\begin{array}{l}\text { increase } \\
\text { (Figure 3a) }\end{array}$ & $\begin{array}{c}\text { negligible } \\
\text { impact } \\
\text { (Figure 4a) } \\
\text { negligible } \\
\text { impact } \\
\text { (Figure 4b) }\end{array}$ & \multirow{2}{*}{$\begin{array}{c}\text { negligible } \\
\text { impact } \\
\text { (Figure 5a) } \\
\text { negligible } \\
\text { impact } \\
\text { (Figure 5b) } \\
\text { negligible } \\
\text { impact } \\
\text { (Figure 5c) }\end{array}$} \\
\hline & $\begin{array}{l}\text { Selection } \\
\text { Entropy }\end{array}$ & $\begin{array}{l}\text { decrease } \\
\text { (Figure 2c) }\end{array}$ & $\begin{array}{l}\text { decrease } \\
\text { (Figure 3c) }\end{array}$ & $\begin{array}{l}\text { decrease } \\
\text { (Figure 4c) }\end{array}$ & \\
\hline & $\begin{array}{c}\text { Network } \\
\text { Centralization }\end{array}$ & $\begin{array}{l}\text { increase } \\
\text { (Figure 2d) }\end{array}$ & $\begin{array}{l}\text { increase } \\
\text { (Figure 3d) }\end{array}$ & $\begin{array}{c}\text { increase } \\
\text { (Figure } 4 \mathrm{~d} \text { ) }\end{array}$ & $\begin{array}{l}\text { increase } \\
\text { (Figure } 5 \mathrm{~d} \text { ) }\end{array}$ \\
\hline
\end{tabular}

\section{Conclusions}

Our work offers some insight on the dynamics of networked societies. The "Death of Distance" [61], due to the World Wide Web, resulted in global interconnectedness. We are interconnected through online social networks, platforms and communities (Facebook, 
LinkedIn, Instagram, Twitter, Airbnb, ResearchGate, TripAdvisor, Booking and YouTube). We are influencing each other, directly or indirectly, intentionally or unintentionally, on global scale. We share knowledge and we rank individuals, services or products. This "ranking" is visible, for example, the Followers at Instagram or Twitter, the Endorsements at LinkedIn, the Likes at Facebook, the 5-star experience at Airbnb or Booking or TripAdvisor, the RG Score at ResearchGate, the Views and Likes at YouTube. Therefore, intelligent decision makers (selecting agents) being aware of this ranking, decide with higher rationality, lowing the selection entropy. Intelligent selections are effectively a mechanism of "preferential attachment" (the newcomer is aware of the degree connectivity of all other nodes and attaches to nodes with higher degree). This behavior increases the centralization of the network society because more and more individuals tend to select the "best option" (high-ranked agents) and the link weights with the low-ranked agents are weakening. Of course, the correlation between the rank/popularity and the quality of the "best option" is not always positive.

\section{Future Work}

Our work is a first step towards the exploration of the emergence of centralization due to the intelligence of agents, communicating in a co-evolving network. We see two main directions for future work, namely, (a) the dependence on the initial network structure (regular, random, small-world, scale-free) and (b) the dependence on time-dependent diagonal weights $w_{\kappa \kappa}(t)$ expressing the agents' innovation capability. Concerning the dependence on the diagonal weights, agents with low innovation capability initially, may eventually develop high innovation capability, which is expected to have significant impact on the emerging dynamics of knowledge inequality, selection entropy and network centralization. More specifically, high network centralization may collapse, due to the emergence of "new" top innovators with high knowledge level. The knowledge of former top innovators becomes depreciated, while the knowledge from "new" top innovators becomes more and more appreciated, due to their higher innovation capability. A real-world paradigm is the mobile phone industry. From 1990s to late 2000s, Nokia and Motorola were both top innovators in the mobile phone industry, with pioneer technology for that time period. However, things changed after 2010, when Samsung and Apple became the "new" top innovators, introducing smartphones like "Samsung Galaxy" and "iPhone".

Author Contributions: Conceptualization, E.I., N.V. and I.A.; methodology, E.I., N.V. and I.A.; software, E.I., N.V. and I.A.; validation, E.I., N.V. and I.A.; formal analysis, E.I., N.V. and I.A.; investigation, E.I., N.V. and I.A.; resources, E.I., N.V. and I.A.; data curation, E.I., N.V. and I.A.; writingoriginal draft preparation, E.I., N.V. and I.A.; writing - review and editing, E.I., N.V. and I.A.; visualization, E.I., N.V. and I.A.; supervision, E.I., N.V. and I.A.; project administration, E.I., N.V. and I.A. All authors have read and agreed to the published version of the manuscript.

Funding: This research received no external funding.

Data Availability Statement: Not applicable.

Acknowledgments: Results presented in this work have been produced using the AUTH Compute Infrastructure and Resources. We appreciate the highly efficient technical support for the computations by the members of the Scientific Computing Office (IT AUTH).

Conflicts of Interest: The authors declare no conflict of interest. 


\section{References}

1. Kirman, A. The economy as an evolving network. J. Evol. Econ. 1997, 7, 339-353. [CrossRef]

2. Antoniou, I.; Reeve, M.; Stenning, V. The Information Society as a Complex System. J. Univers. Comput. Sci. 2000, 6, $272-288$. [CrossRef]

3. Schweitzer, F.; Fagiolo, G.; Sornette, D.; Vega-Redondo, F.; Vespignani, A.; White, D.R. Economic Networks: The New Challenges. Science 2009, 325, 422-425. [CrossRef] [PubMed]

4. Castells, M. The Rise of the Network Society (The Information Age: Economy, Society, and Culture), 2nd ed.; Wiley-Blackwell: Hoboken, NJ, USA, 2000; Volume 1.

5. Hansen, M.T. Knowledge Networks: Explaining Effective Knowledge Sharing in Multiunit Companies. Organ. Sci. 2002, 13, 232-248. [CrossRef]

6. Wang, S.; Noe, R.A. Knowledge sharing: A review and directions for future research. Hum. Resour. Manag. Rev. 2010, 20, 115-131. [CrossRef]

7. Phelps, C.C.; Heidl, R.; Wadhwa, A. Knowledge, Networks, and Knowledge Networks. J. Manag. 2012, 38, 1115-1166. [CrossRef]

8. Ioannidis, E.; Varsakelis, N.; Antoniou, I.; Evangelos, I.; Nikos, V.; Ioannis, A. False Beliefs in Unreliable Knowledge Networks. Phys. A Stat. Mech. Its Appl. 2017, 470, 275-295. [CrossRef]

9. Ioannidis, E.; Varsakelis, N.; Antoniou, I. Communication Policies in Knowledge Networks. Phys. A Stat. Mech. Its Appl. 2018, 492, 360-374. [CrossRef]

10. Ioannidis, E.; Varsakelis, N.; Antoniou, I. Experts in Knowledge Networks: Central Positioning and Intelligent Selections. Phys. A Stat. Mech. Its Appl. 2018, 509, 890-905. [CrossRef]

11. Ioannidis, E. Modeling Knowledge Networks. Ph.D. Thesis, Aristotle University of Thessaloniki, Thessaloniki, Greece, 2018. Available online: https://ikee.lib.auth.gr/record/297396/files/GRI-2018-21354.pdf (accessed on 20 November 2020).

12. Cowan, R.; Jonard, N. The dynamics of collective invention. J. Econ. Behav. Organ. 2003, 52, 513-532. [CrossRef]

13. Cowan, R.; Jonard, N. Network structure and the diffusion of knowledge. J. Econ. Dyn. Control 2004, 28, 1557-1575. [CrossRef]

14. Kim, H.; Park, Y. Structural effects of R\&D collaboration network on knowledge diffusion performance. Expert Syst. Appl. 2009, 36, 8986-8992. [CrossRef]

15. Tang, F.; Xi, Y.; Ma, J. Estimating the effect of organizational structure on knowledge transfer: A neural network approach. Expert Syst. Appl. 2006, 30, 796-800. [CrossRef]

16. Tang, F.; Mu, J.; MacLachlan, D.L. Disseminative capacity, organizational structure and knowledge transfer. Expert Syst. Appl. 2010, 37, 1586-1593. [CrossRef]

17. Lin, M.; Li, N. Scale-free network provides an optimal pattern for knowledge transfer. Phys. A Stat. Mech. Its Appl. 2010, 389, 473-480. [CrossRef]

18. Xuan, Z.; Xia, H.; Du, Y. Adjustment of knowledge-connection structure affects the performance of knowledge transfer. Expert Syst. Appl. 2011, 38, 14935-14944. [CrossRef]

19. Xia, H.; Du, Y.; Xuan, Z. Structural Evolution in Knowledge Transfer Network: An Agent-Based Model. In Artificial Intelligence: Foundations, Theory, and Algorithms; Springer Science and Business Media LLC: Berlin/Heidelberg, Germany, 2013; Volume 424, pp. 31-38.

20. Luo, S.; Du, Y.; Liu, P.; Xuan, Z.; Wang, Y. A study on coevolutionary dynamics of knowledge diffusion and social network structure. Expert Syst. Appl. 2015, 42, 3619-3633. [CrossRef]

21. Jian-Guo, L.; Zhou, Q.; Guo, Q.; Yang, Z.-H.; Xie, F.; Han, J.-T. Knowledge diffusion of dynamical network in terms of interaction frequency. Sci. Rep. 2017, 7, 10755. [CrossRef]

22. Liu, J.-G.; Yang, G.-Y.; Hu, Z.-L. A Knowledge Generation Model via the Hypernetwork. PLoS ONE 2014, 9, e89746. [CrossRef] [PubMed]

23. Yang, G.-Y.; Hu, Z.-L.; Liu, J.-G. Knowledge diffusion in the collaboration hypernetwork. Phys. A Stat. Mech. Appl. 2015, 419, 429-436. [CrossRef]

24. Zhang, H.; Wu, W.; Zhao, L. A study of knowledge supernetworks and network robustness in different business incubators. Phys. A Stat. Mech. Appl. 2016, 447, 545-560. [CrossRef]

25. Simon, H.A. A Behavioral Model of Rational Choice. Q. J. Econ. 1955, 69. [CrossRef]

26. Simon, H.A. Rational Decision Making in Business Organizations. Am. Econ. Rev. 1979, 69, 493-513. Available online: http: //www.jstor.org/stable/1808698 (accessed on 20 November 2020).

27. Wang, J.-P.; Guo, Q.; Yang, G.-Y.; Liu, J.-G. Improved knowledge diffusion model based on the collaboration hypernetwork. Phys. A Stat. Mech. Appl. 2015, 428, 250-256. [CrossRef]

28. Lin, M.; Wei, J. The impact of innovation intermediary on knowledge transfer. Phys. A Stat. Mech. Appl. 2018, 502, 21-28. [CrossRef]

29. Ioannidis, E.; Varsakelis, N.; Antoniou, I. Promoters versus Adversaries of Change: Agent-Based Modeling of Organizational Conflict in Co-Evolving Networks. Mathematics 2020, 8, 2235. [CrossRef]

30. Wi, H.; Oh, S.; Mun, J.; Jung, M. A team formation model based on knowledge and collaboration. Expert Syst. Appl. 2009, 36, 9121-9134. [CrossRef]

31. Gladstones, W.H.; Regan, M.A.; Lee, R.B. Division of Attention: The Single-Channel Hypothesis Revisited. Q. J. Exp. Psychol. Sect. A 1989, 41, 1-17. [CrossRef] 
32. Pashler, H. Dual-task interference in simple tasks: Data and theory. Psychol. Bull. 1994, 116, 220-244. [CrossRef]

33. Indermühle, R.; Troche, S.; Rammsayer, T. Personality and the psychological refractory period: No evidence for an extraversionor intelligence-related effect. Can. J. Behav. Sci. Rev. Can. des Sci. du Comport. 2011, 43, 214-221. [CrossRef]

34. Cowan, R.; Jonard, N.; Özman, M. Knowledge dynamics in a network industry. Technol. Forecast. Soc. Chang. 2004, 71, 469-484. [CrossRef]

35. Knuth, D.E. Two Notes on Notation. Am. Math. Mon. 1992, 99, 403-422. [CrossRef]

36. Zhao, L.; Zhang, H.; Wu, W. Knowledge service decision making in business incubators based on the supernetwork model. Phys. A Stat. Mech. Appl. 2017, 479, 249-264. [CrossRef]

37. Ioannidis, E.; Varsakelis, N.; Antoniou, I. Change agents and internal communications in organizational networks. Phys. A Stat. Mech. Appl. 2019, 528, 121385. [CrossRef]

38. Havlin, S.; Ben-Avraham, D. Diffusion in disordered media. Adv. Phys. 1987, 36, 695-798. [CrossRef]

39. Skarpalezos, L.; Kittas, A.; Argyrakis, P.; Cohen, R.; Havlin, S. Anomalous biased diffusion in networks. Phys. Rev. E 2013, 88, 012817-012823. [CrossRef]

40. Wang, H.; Chen, C.; Qu, B.; Li, D.; Havlin, S. Epidemic mitigation via awareness propagation in communication networks: The role of time scales. New J. Phys. 2017, 19, 073039. [CrossRef]

41. Zhuang, E.; Chen, G.; Feng, G. A network model of knowledge accumulation through diffusion and upgrade. Phys. A Stat. Mech. Appl. 2011, 390, 2582-2592. [CrossRef]

42. Graupe, D. Principles of Artificial Neural Networks, 3rd ed.; World Scientific: Singapore, 2013.

43. Fan, K.; Pedrycz, W. Opinion evolution influenced by informed agents. Phys. A Stat. Mech. Appl. 2016, 462, 431-441. [CrossRef]

44. Zhu, H.-M.; Zhang, S.-T.; Jin, Z. The effects of online social networks on tacit knowledge transmission. Phys. A Stat. Mech. Appl. 2016, 441, 192-198. [CrossRef]

45. Gladwell, M. The Tipping Point: How Little Things Can Make a Big Difference; Hachette Book Group: New York, NY, USA, 2002.

46. Dunbar, R. How Many Friends Does One Person Need? Dunbar's Number and Other Evolutionary Quirks; Harvard University Press: Cambridge, MA, USA, 2011.

47. Tur, E.M.; Azagra-Caro, J.M. The coevolution of endogenous knowledge networks and knowledge creation. J. Econ. Behav. Organ. 2018, 145, 424-434. [CrossRef]

48. Morone, P.; Taylor, R. Knowledge diffusion dynamics and network properties of face-to-face interactions. J. Evol. Econ. 2004, 14, 327-351. [CrossRef]

49. Cremonini, M. Introducing serendipity in a social network model of knowledge diffusion. Chaos Solitons Fractals 2016, 90 , 64-71. [CrossRef]

50. Freeman, L.C. Centrality in social networks conceptual clarification. Soc. Netw. 1978, 1, 215-239. [CrossRef]

51. Angelidis, G.; Ioannidis, E.; Makris, G.; Antoniou, I.; Varsakelis, N. Competitive Conditions in Global Value Chain Networks: An Assessment Using Entropy and Network Analysis. Entropy 2020, 22, 1068. [CrossRef]

52. Thurner, S.; Klimek, P.; Hanel, R. Introduction to the Theory of Complex Systems; Oxford University Press (OUP): Oxford, UK, 2018.

53. Zimmermann, M.G.; Eguíluz, V.M.; Miguel, M.S. Coevolution of dynamical states and interactions in dynamic networks. Phys. Rev. E 2004, 69, 065102. [CrossRef]

54. Vazquez, F.; Eguíluz, V.M.; Miguel, M.S. Generic Absorbing Transition in Coevolution Dynamics. Phys. Rev. Lett. 2008, 100, 108702. [CrossRef]

55. Biely, C.; Hanel, R.; Thurner, S. Socio-economical dynamics as a solvable spin system on co-evolving networks. Eur. Phys. J. B 2008, 67, 285-289. [CrossRef]

56. Diakonova, M.; Eguíluz, V.M.; Miguel, M.S. Noise in coevolving networks. Phys. Rev. E 2015, 92, 032803. [CrossRef]

57. Min, B.; Miguel, M.S. Fragmentation transitions in a coevolving nonlinear voter model. Sci. Rep. 2017, 7, 1-9. [CrossRef]

58. Raducha, T.; Min, B.; Miguel, M.S. Coevolving nonlinear voter model with triadic closure. EPL Europhys. Lett. 2018, 124, 1-6. [CrossRef]

59. Kurzweil, R. The Age of Spiritual Machines: When Computers Exceed Human Intelligence; Viking Press: New York, NY, USA, 1999.

60. Pan, R.K.; Petersen, A.M.; Pammolli, F.; Fortunato, S. The memory of science: Inflation, myopia, and the knowledge network. J. Inf. 2018, 12, 656-678. [CrossRef]

61. Cairncross, F. The Death of Distance: How the Communications Revolution Is Changing our Lives; Harvard University Press: Cambridge, MA, USA, 2001. 